\title{
Fatty acid compositions of fish oil extracted from different parts of Indian mackerel (Rastrelliger kanagurta) using various techniques of supercritical $\mathrm{CO} 2$ extraction.
}

\begin{abstract}
Fatty acid compositions of fish oil extracted from different parts of Indian mackerel (Rastrelliger kanagurta) using various techniques of supercritical carbon dioxide (SC-CO2) at optimised conditions ( $35 \mathrm{MPa}, 60{ }^{\circ} \mathrm{C}, 2 \mathrm{ml} / \mathrm{min}$ ) were analysed and compared to the results of Soxhlet extraction. The amount of polyunsaturated fatty acids (PUFA) recovered (as a percentage of total extracted fatty acids) were within the ranges of 73.24-74.68\% in the skin, $68.36-69.37 \%$ in the flesh, $56.20-57.3 \%$ in the viscera and $61.21-62.09 \%$ in the heads. The greatest amount of the $\omega-3$ fatty acids, especially eicosapentaenoic acid (EPA) and docosahexaenoic acid (DHA), were found in fish skin followed by flesh, heads and viscera. The greatest amounts of EPA (9-12\%) and DHA (10-14\%) were obtained using the soaking and pressure swing techniques. The pressure swing and soaking techniques are the most effective techniques for extracting the $\omega-3$ family of fatty acids from fish samples.
\end{abstract}

Keyword: PUFA; $\omega-3$ Fatty acids; Indian mackerel; Supercritical carbon dioxide extraction. 